ESAIM: PROCEEDINGS, December 2013, Vol. 43, p. 95-107

S. Descombes, B. Dussoubs, S. Faure, L. Gouarin, V. Louvet, M. Massot, V. Miele, Editors

\title{
EXTENDED GENERALIZED LAGRANGIAN MULTIPLIERS FOR MAGNETOHYDRODYNAMICS USING ADAPTIVE MULTIRESOLUTION METHODS
}

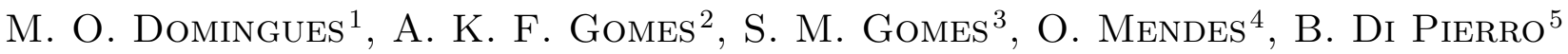 \\ ET K. SCHNEIDER ${ }^{6}$
}

Résumé. Nous présentons une nouvelle méthode de multirésolution adaptative pour la simulation numérique de la magnétohydrodynamique idéale. Les équations qui régissent la dynamique, i.e., les équations d'Euler compressible couplées aux équations de Maxwell sont discrétisées suivant un schéma de type volumes finis sur un maillage cartésien en deux dimensions. L'adaptativité en espace est obtenue en utilisant une analyse de multirésolution en moyenne de cellule proposée par Harten, qui est une méthode fiable pour le raffinement local du maillage tout en contrôlant l'érreur. La discrétisation temporelle est un schéma de Runge-Kutta qui intègre un contrôle automatique du pas de temps. Pour imposer l'incompressibilié du champs magnétique, une approche par multiplicateur de Lagrange généralisé est utilisée, ici de type parabolique-hyperbolique. Pour illustrer les capacitées de cette méthode, des applications à des problèmes de Riemann ont été réalisées. Les coûts en mémoire sont présentés, et la précision de la méthode est évaluée par comparaison avec les solutions exactes du problème.

\begin{abstract}
We present a new adaptive multiresoltion method for the numerical simulation of ideal magnetohydrodynamics. The governing equations, i.e., the compressible Euler equations coupled with the Maxwell equations are discretized using a finite volume scheme on a two-dimensional Cartesian mesh. Adaptivity in space is obtained via Harten's cell average multiresolution analysis, which allows the reliable introduction of a locally refined mesh while controlling the error. The explicit time discretization uses a compact Runge-Kutta method for local time stepping and an embedded Runge-Kutta scheme for automatic time step control. An extended generalized Lagrangian multiplier approach with the mixed hyperbolic-parabolic correction type is used to control the incompressibility of the magnetic field. Applications to a two-dimensional problem illustrate the properties of the method. Memory savings and numerical divergences of magnetic field are reported and the accuracy of the adaptive computations is assessed by comparing with the available exact solution.
\end{abstract}

\footnotetext{
1 Laboratório Associado de Computação e Matemática Aplicada (LAC), Coordenadoria dos Laboratórios Associados (CTE), Instituto Nacional de Pesquisas Espaciais (INPE), Av. dos Astronautas 1758, 12227-010 São José dos Campos, São Paulo, Brazil (e-mail : margarete@lac.inpe.br, margarete.oliveira.domingues@gmail.com)

2 Pós Graduação em Computação Aplicada, CTE, INPE (e-mail : annakfg@gmail.com)

3 Universidade Estadual de Campinas (Unicamp), IMECC, Rua Sérgio Buarque de Holanda, 651, Cidade Universitária Zeferino Vaz, 13083-859 Campinas, São Paulo, Brazil (e-mail : soniag@ime.unicamp.br)

4 Divisão de Geofísica Espacial(DGE), Coordenação de Ciências Espaciais(CEA), INPE (e-mail : odim@dge.inpe.br)

${ }^{5}$ IRPHE-CNRS, Aix-Marseille Université, 49 rue F. Joliot-Curie, 13453 Marseille Cedex 13, France (e-mail : dipierro@irphe .univ-mrs.fr)

6 M2P2-CNRS \& Centre de Mathématiques et d'Informatique (CMI), Aix-Marseille Université, 38 rue F. Joliot-Curie, 13451

Marseille Cedex 20, France (e-mail : kschneid@cmi.univ-mrs.fr)
}

(C) EDP Sciences, SMAI 2013 


\section{INTRODUCTION}

Plasmas and electrically conducting fluids, ubiquitous in our daily life, are of major importance, as for example, in the Sun, which strongly influences the magnetic field around Earth, or for the dynamo action inside the liquid metal of Earth. The numerical simulation of magnetohydrodynamics (MHD) modeling such phenomena encounters, in comparison to ordinary hydrodynamics, additional complexities which are not only due to the presence of multiple spatial and temporal scales. The nonlocal character of boundary conditions of the magnetic field requires specific approaches, like matching the magnetic field at the boundary with the field in a surrounding vacuum, and in particular, the incompressibility of the magnetic field necessitates precise and efficient numerical techniques. Divergence errors in the magnetic field modify the underlying physics of the problem. Traditionally, Helmholtz decompositions, also called Hodge decompositions, are used to project the magnetic field onto divergence free fields. However, this approach is computationally expensive as it requires the solution of a Poisson equation, which implies the solution of very large linear systems especially in 3D. For a discussion on this topic we refer the reader e.g. to $[2,3,20,23]$. Divergence cleaning techniques based on Lagrange multipliers have been introduced in the finite element context by Assous et al. [1] for time-dependent Maxwell equations. Since then different developments leading to various types of approaches can be found in the literature [2,3,19,23]. Munz et al. [18] introduced a generalized Lagrangian multiplier formulation of Maxwell equations, which leads to different PDEs for the multiplier, being either of hyperbolic, parabolic, elliptic or mixed types. In the current work we use a variant of the previously cited approaches, the Extended Generalized Lagrange Multiplier (EGLM) designed by Dedner et al. [5] with a mixed parabolic-hiperbolic correction. The idea of the latter technique is not to enforce the divergence free condition exactly, but to promote a natural evolution of the system toward a divergence free state as discussed in [15].

The adaptive method of the present paper falls into the multiresolution (MR) category, which is designed to speed up finite volume schemes for time dependent conservation laws, based on ideas originally introduced in the work of Harten $[13,14]$. The MR method is also combined with time adaptive strategies using either local or controlled time stepping. A review of multiresolution techniques can be found in the book of Müller [17], or in the review [10] and references therein.

The aim of this paper is to combine for the first time the divergence cleaning technique for the magnetic field introduced by Dedner et al. [5] with adaptive multiresolution computations and to check its feasibility interplaying with adaptivity. The adaptive multiresolution code originally developed by Roussel et al. [21] has been extended to include Maxwell equations governing the magnetic field [12]. For divergence cleaning the EGLM formulation is used. The resulting new method is applied to a Riemann test problem for which the exact solution is known and the divergence of the magnetic field remains zero. The accuracy of the adaptive computations is assessed and their efficiency in terms of memory compression compared to a finite volume scheme on a regular grid is reported.

The paper is organized as follows: after a presentation of the governing MHD equations in Section 1, we recall the EGLM formulation in Section 2. In Section 3 space and time discretizations are briefly described together with the EGLM discretization with the mixed hyperbolic-parabolic correction. In Section 4, numerical results are presented. Finally, some remarks and perspectives for further development of the method are presented.

\section{MHD Ideal Equations}

The ideal magnetohydrodynamics equations are a mathematical model for the interaction of a compressible, inviscous and eletrically conducting fluid and a magnetic field. This model is derived from the Euler equations of gas dynamics coupled with the Maxwell equations, which gives an additional evolutionary equation for the magnetic field, and a divergence-free constraint based on Gauss' law. The ideal two-dimensional MHD system 
is given by the following equations

$$
\begin{array}{rlrl}
\frac{\partial \rho}{\partial t}+\nabla \cdot(\rho \mathbf{u}) & =0, & & \text { (Mass conservation) } \\
\frac{\partial E}{\partial t}+\nabla \cdot\left[\left(E+p+\frac{\mathbf{B} \cdot \mathbf{B}}{2}\right) \mathbf{u}-(\mathbf{u} \cdot \mathbf{B}) \mathbf{B}\right] & =0, & & \text { (Energy conservation) } \\
\frac{\partial \rho \mathbf{u}}{\partial t}+\nabla \cdot\left[\rho \mathbf{u u}+\left(p+\frac{\mathbf{B} \cdot \mathbf{B}}{2}\right) \mathbf{I}-\mathbf{B B}\right] & =\mathbf{0}, & \text { (Momentum conservation) } \\
\frac{\partial \mathbf{B}}{\partial t}+\nabla \cdot(\mathbf{u B}-\mathbf{B u}) & =\mathbf{0} & \text { (Induction equation) },
\end{array}
$$

together with

$$
\nabla \cdot \mathbf{B}=\mathbf{0} \quad \text { (Divergence constraint) }
$$

where $\rho$ represents density, $p$ is the pressure, $\mathbf{u}=\left(v_{x}, v_{y}, v_{z}\right)$ is the velocity vector, $\mathbf{B}=\left(B_{x}, B_{y}, B_{z}\right)$ is the magnetic field vector, $\mathbf{I}$ is the identity tensor of order 2 , and $\gamma$ is the adiabatic constant $(\gamma>1)$. The pressure is given by the constitutive law $p=(\gamma-1)\left(E-\rho \frac{\mathbf{u} \cdot \mathbf{u}}{2}-\frac{\mathbf{B} \cdot \mathbf{B}}{2}\right)$. The above system is completed by suitable initial and boundary conditions. Nevertheless, the induction equation can be rewritten as $\frac{\partial \mathbf{B}}{\partial t}+\nabla \times(\mathbf{B} \times \mathbf{u})=\mathbf{0}$. Therefore, applying the divergence operator yields $\frac{\partial}{\partial t}(\nabla \cdot \mathbf{B})=0$, as $\nabla \cdot(\nabla \times) \equiv 0$, which shows that if the initial condition of the magnetic field is divergence-free it will remain divergence-free during time evolution. However, numerically, the incompressibility of the magnetic field is not always preserved, and thus, non-physical results could be obtained or the computations may even become unstable [3].

Since the 1980s usual numerical MHD methodologies consider the enforcement of the physical property of the divergence-free constraint. In the context of this study, having in mind the application of a multiresolution method using explicit time integration, we adopt EGLM-MHD with the mixed parabolic-hyperbolic correction described in the following section.

\section{IDEAL MHD EQUATIONS WITH EGLM}

The Extended Generalized Lagrangian Multiplier conservative system formulation with the hyperbolicparabolic correction was proposed in Dedner et al. [5]. It fits well in a pre-existing MHD model by introducing an additional scalar field $\psi$, which couples the divergence constraint equation (Eq. 2) to Faraday's law, modifying the induction equation (Eq. 1d). Moreover, some source terms are added similary to what was proposed in [19]. The mixed hyperbolic-parabolic correction introduces two parameters, one related to the hyperbolic correction, namely $c_{h}$, to propagate the divergence errors, and another one, related to the parabolic correction $c_{p}$, to control the rate at which monopoles are damped in the new divergence constraint equation and a source term in the new divergence constraint equation.

The remaining terms in the equations are not changed. The conservative characteristic of this system is not lost if one just uses the standard Generalized Lagrangian Multiplier conservative system (GLM) approach, i.e., if we do not consider the source terms. Here we are not presenting the results for this case, as they are similar to EGLM-MHD. 
The resulting EGLM-MHD equations with hyperbolic-parabolic correction are written in dimensionless form [5]

$$
\begin{aligned}
\frac{\partial \rho}{\partial t}+\nabla \cdot(\rho \mathbf{u}) & =0, \\
\frac{\partial E}{\partial t}+\nabla \cdot\left[\left(E+p+\frac{\mathbf{B} \cdot \mathbf{B}}{2}\right) \mathbf{u}-\mathbf{B}(\mathbf{u} \cdot \mathbf{B})\right] & =-\mathbf{B} \cdot(\nabla \psi), \\
\frac{\partial(\rho \mathbf{u})}{\partial t}+\nabla \cdot\left[\rho \mathbf{u u}+\left(p+\frac{\mathbf{B} \cdot \mathbf{B}}{2}\right) \mathbf{I}-\mathbf{B B}\right] & =-(\nabla \cdot \mathbf{B}) \mathbf{B}, \\
\frac{\partial \mathbf{B}}{\partial t}+\nabla \cdot[\mathbf{u B}-\mathbf{B u}+\psi \mathbf{I}] & =0, \\
\frac{\partial \psi}{\partial t}+c_{h}^{2} \nabla \cdot \mathbf{B} & =-\frac{c_{h}^{2}}{c_{p}^{2}} \psi .
\end{aligned}
$$

where the parameter $c_{h}>0$. This parameter has a strong influence on the correction. In [5] different choices for the parameter $c_{h}$ and $c_{p}$ were suggested and discussed, for example $c_{h}=c_{h}(t):=c_{C F L} \frac{\min (d x, d y)}{d t}, c_{C F L} \in(0,1)$. A fixed ratio $c_{p}^{2} / c_{h}=0.18$, independent of the resolution, was also suggested. For futher details and other choices we refer to $[6,24]$. Here we use the resolution independent value $c_{p}^{2} / c_{h}=0.18$.

Considering the vector of convervatives quantities $\mathbf{Q}=(\rho, E, \rho \mathbf{u}, \mathbf{B}, \psi)$ the EGLM-MHD system can be written in the compact form

$$
\frac{\partial \mathbf{Q}}{\partial t}+\nabla \cdot \mathbf{F}(\mathbf{Q})=\mathbf{S}(\mathbf{Q})
$$

where $\mathbf{F}$ denotes the physical fluxes and $\mathbf{S}$ contains all source terms.

\section{Space-time adaptive strategies}

Applying a Finite Volume (FV) discretization to the compact form of the EGLM-MHD system (Eq. 4) results in an Ordinary Differential Equation (ODE) system of the form

$$
\frac{d \overline{\mathbf{Q}}}{d t}=-\mathbf{F}(\overline{\mathbf{Q}})+\mathbf{S}(\overline{\mathbf{Q}})
$$

where $\overline{\mathbf{Q}}(t)$ denotes approximated cell-averages of the quantities on the cells $C$ forming a partition of the computational domain, $\mathbf{F}(\overline{\mathbf{Q}})$ is the numerical flux, and $\mathbf{S}(\overline{\mathbf{Q}})$ is the numerical source term. Approximate solutions $\overline{\mathbf{Q}}^{n}$ at a sequence of time instants $t^{n}$ are obtained using an explicit ODE solver. For time integration, explicit Runge-Kutta schemes of second and third order are used. The initial value of $\psi$ is zero. The resulting FV reference scheme follows the steps:

- The parameter $c_{h}$ is computed.

- In each step of the time integration scheme:

(1) A spliting method is used.

(2) The homegeneous GLM system is solved:

- The normal component of the magnetic field $B_{N}$ in the flux direction (Eq. 3d) and the divergence constraint equation (Eq. 3e) are decoupled from the other variables. These two equations form a linear system and a local Riemann problem can be compute directly, where the numerical flux is $\left(\psi, c_{h}^{2} B_{N}\right)$ for $B_{N}$ and $\psi$, respectively. The same is done in the other direction, in the two-dimensional case.

- For the remaining variables we compute the numerical flux using HLLD scheme with Davis limiter [16]. 
- The computed values of $\psi$ are used to update the mixed correction source term for $\psi^{n+1}$, computing $\psi^{n+1}=\exp \left(-\Delta t^{n} \frac{c_{h}^{2}}{c p^{2}}\right) \psi$

- The other source terms are added.

The adaptive method of the present paper falls into the MR category, which is designed to speed up finite volume schemes for time dependent conservation laws. The MR method is also combined with time adaptive strategies using either local or controlled time stepping. In the following, a brief summary of these techniques is given. For a detailed description of these combined space-time adaptive strategies, we refer to [8-11,21].

\subsection{MR Scheme}

The main idea behind MR schemes is to use the decay of wavelet coefficients of the numerical solution to obtain information on the local regularity of the solution. Small coefficients occur in regions of smoothness of the solution, where coarser grids can be used, while fine grid refinement is only applied in regions where the coefficients are significant, corresponding to strong variations [4]. Adaptive MR representations are obtained by stopping the refinement in a cell at a certain scale level, where the wavelet coefficients are non-significant. In the finite volume context, instead of using a cell-average representation on the uniform fine mesh, the MR scheme computes the numerical solution represented by cell-averages on an adaptive sparse mesh, which is formed by the cells whose wavelet coefficients are significant and above a given threshold. Localized structures in MHD solutions, such as discontinuities or shocks, could appear in different space positions in different variables, and thus, the adaptive mesh of the MHD system is a union of the individual adaptive meshes, as presented in the example of Fig. 1.

A natural way to store the reduced MR data is to use a tree data structure, where grid adaptivity is related with an incomplete tree, and where the refinement may be interrupted at intermediate scale levels. This means that, using the tree terminology, a MR grid is formed by leaves, which are nodes without children. These leaves are related to the cell, which indeed will be evolved in time.

In general, there are three basic steps in the application of a MR scheme: refinement, evolution, and coarsening. The refinement operator is a precautionary measure to account for a possible translation of the solution or the creation of finer scales in the solution between two subsequent time steps. Since the regions of smoothness or irregularities of the solution may change with time, the MR grid at $t^{n}$ may not be convenient anymore at the next time step $t^{n+1}$. Therefore, before doing the time evolution, the representation of the solution should be interpolated onto an extended grid that is expected to be a refinement of the adaptive grid at $t^{n}$, and to contain the adaptive grid at $t^{n+1}$. After that, the time evolution operator is applied on the leaves of the extended grid. To compute numerical fluxes between cells of different levels, we also add extra cells, called virtual leaves, that will not be used in the time evolution. Conservation is ensured by the fact that the fluxes are always computed on the higher level, the value being projected to the leaves of a lower level. Then, a wavelet thresholding operation (coarsening) is applied in order to unrefine the cells in the extended grid that are not necessary for an accurate representation of the solution at $t^{n+1}$. This data compression is based on the definition of deletable cells, where the wavelet coefficients are not significant, i.e., their magnitudes are bellow a threshold parameter $\epsilon_{\ell}$, where $\ell$ denotes the cell scale level.

In order to control the $L^{1}$-norm, Harten's thresholding strategy is used, for which

$$
\epsilon^{\ell}=\frac{\epsilon_{0}}{|\Omega|} 2^{d(\ell-L+1)}, \quad 0 \leq \ell \leq L-1
$$

where $d=2$ is the space dimension, $L$ is the finest scale level, and $|\Omega|$ is the area of the domain. For comparison, we shall also consider level independent threshold parameters: $\epsilon_{\ell} \equiv \epsilon$, for all $1 \leq \ell \leq L-1$.

For the applications of the present paper, the multiresolution analysis corresponds to a prediction operator based on a third order polynomial interpolation on the cell-averages. The time integration is performed by a second order Runge-Kutta scheme combined with local time steping (MR/LT), or an embbeded Runge-Kutta $2(3)$ for automatic time step control (MR/CT). 
(a)

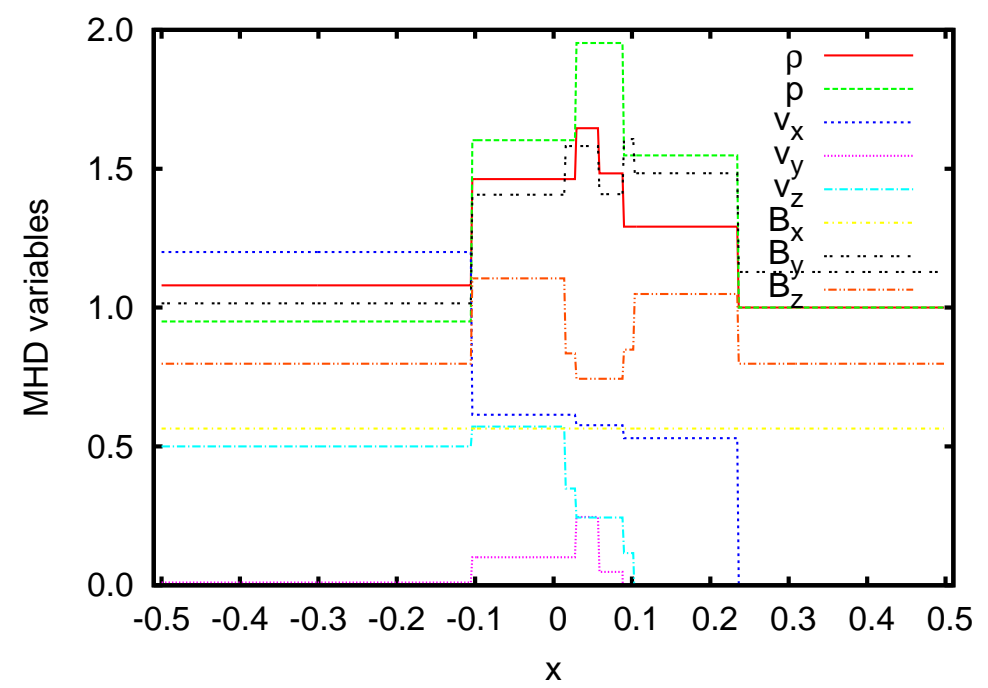

(b)

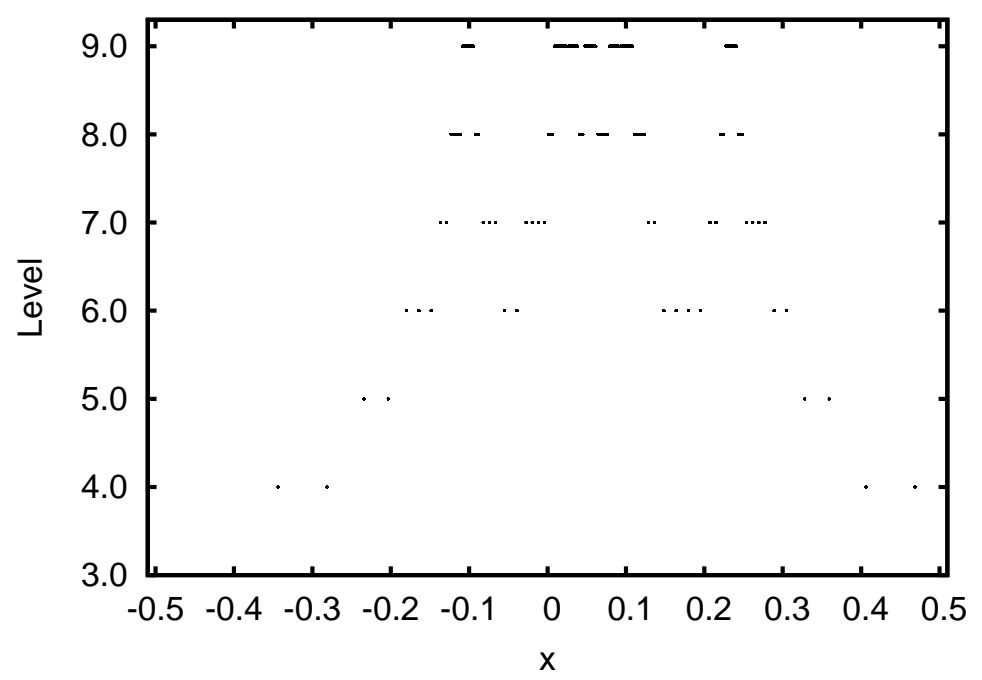

Figure 1. One-dimension representation of an exact solution of the dimensionless MHD variables: (a) density, pressure, velocity and magnetic field; and (b) corresponding adaptive mesh in the level $\times x$ plane constructed with a constant threshold parameter $\epsilon=0.001$.

\subsection{MR/LT Scheme}

In order to save CPU time, instead of evolving the solution with a single time step on all grid cells, the solution may be integrated with a different time step for each level, as proposed in [8]. For the MR/LT scheme, the time step $\Delta t^{n}$ at the finest scale level $L$ is determined by the CFL condition. The principle is then to evolve the cells at lower levels $0 \leq \ell<L$ with larger time steps $\Delta t_{\ell}^{n}=2^{L-\ell} \Delta t^{n}$. The required missing values in ghost cells are interpolated at intermediate time levels. 


\subsection{MR/CT Scheme}

In the $\mathrm{MR} / \mathrm{CT}$ scheme, the time integration is performed with a global but variable time step $\Delta t$, whose size is chosen dynamically. The MR/CT scheme adopts a strategy from ODE simulations, where the time step size selection is based on the estimated local truncation error. The main reason for controlling the error in the solution is to obtain an accurate and safe integration in the whole interval. To make the integration more

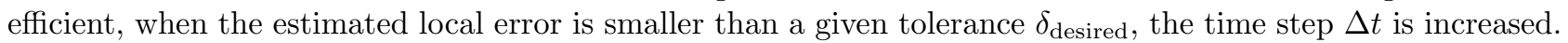
On the other hand, if the local error increases, the time step is decreased. It may happen that a new time step is larger than the limit prescribed by the CFL condition. For the applications of the present paper, the MR/CT scheme is based on the embedded Runge-Kutta Fehlberg 2(3) ODE solver [9]. The initial time step is determined by an input $C F L(0)$ parameter provided by the user. From one time instant to the next one, given the current $\Delta t$, the next $\Delta t_{n e w}$ is determined to maintain the local truncation error below $\delta_{\text {desired }}$. Precisely, it has the form $\Delta t_{n e w}=\xi \Delta t$, with

$$
\xi=\left[\frac{\delta_{\text {desired }}}{\left|\overline{\mathbf{Q}}_{(R K 2)}-\overline{\mathbf{Q}}_{(R K 3)}\right|}\right]^{1 / 3} .
$$

where $\overline{\mathbf{Q}}_{(R K 2)}$ and $\overline{\mathbf{Q}}_{(R K 3)}$ stand for the solutions produced by second and third order Runge-Kutta schemes. To prevent the time step of varying too abruptly or to be sure that $\Delta t_{n e w}$ in fact will produce an error less than $\delta_{\text {desired }}$, the time step variation is limited by a factor that decreases exponentially from $10 \%$, in the initial time step, to $1 \%$ after few iterations.

The reference EGLM-MHD FV code used in this work has been developed in [12] in $C^{++}$language, inspired by the Fortran code developed by [7], including an upgrade and new features for the implementation of the numerical flux HLLD. The EGLM-MHD-MR code developed in [12] has been implemented in the CARMEN code [21], which nowadays also includes automatic time step control and local time adaptivity, as described in $[8,9,11]$.

\section{Numerical Tests}

To verify the code and to analyze the numerical effects that MR schemes could have on the divergence cleaning of the magnetic field, we study in this paper an example of an initial condition similar to a 1D Riemann problem, in a 2D MHD code. The initial condition is given in Table 1 and the exact solution at time $t=0.1$ in Table 2. The latter is computed based on the algorithms described in [22], with the program available at https://web.mathcces.rwth-aachen.de/mhdsolve/.

TABLE 1. 1D Riemann like initial condition, for the $2 \mathrm{D}$ domain $[-0.5,0.5] \times[-0.5,0.5]$.

\begin{tabular}{c|c|c|c|c|c|c|c|c|c}
\hline \hline & $\rho$ & $p$ & $v_{x}$ & $v_{y}$ & $v_{z}$ & $B_{x}$ & $B_{y}$ & $B_{z}$ & $\psi$ \\
\hline$x \leq 0$ & 1.08 & 0.95 & 1.20 & 0.01 & 0.50 & $1.00 / \sqrt{\pi}$ & $1.80 / \sqrt{\pi}$ & $2.00 / \sqrt{2 \pi}$ & 0.00 \\
$x>0$ & 1.00 & 1.00 & 0.00 & 0.00 & 0.00 & $1.00 / \sqrt{\pi}$ & $2.00 / \sqrt{\pi}$ & $2.00 / \sqrt{2 \pi}$ & 0.00 \\
\hline \hline
\end{tabular}

NOTE: Adapted from [5]. Values of the magnetic field $\mathbf{B}$ have a different normalization to be adequate to the computed exact solution, and $\gamma=\frac{5}{3}$. 
TABLE 2. Exact solution of the MHD system at time $t=0.1$, for $y \in[-0.5,0.5]$.

\begin{tabular}{r|r|r|r|r|r|r|r|r}
\hline \hline$x$ interval & $\rho$ & $p$ & $v_{x}$ & $v_{y}$ & $v_{z}$ & $B_{x}$ & $B_{y}$ & $B_{z}$ \\
\hline$[-0.5000,-0.1040)$ & 1.0800 & 0.9500 & 1.2000 & 0.0100 & 0.5000 & $1 / \sqrt{\pi}$ & 1.0155 & 0.7979 \\
{$[-0.1040,0.0147)$} & 1.4626 & 1.6026 & 0.6139 & 0.1011 & 0.5716 & $1 / \sqrt{\pi}$ & 1.4064 & 1.1050 \\
{$[0.0147,0.0276)$} & 1.4626 & 1.6026 & 0.6139 & 0.1011 & 0.3484 & $1 / \sqrt{\pi}$ & 1.5816 & 0.8351 \\
{$[0.0276,0.0576)$} & 1.6459 & 1.9526 & 0.5763 & 0.2460 & 0.2440 & $1 / \sqrt{\pi}$ & 1.5816 & 0.7436 \\
{$[0.0576,0.0888)$} & 1.4832 & 1.9526 & 0.5763 & 0.0482 & 0.2440 & $1 / \sqrt{\pi}$ & 1.4083 & 0.7436 \\
{$[0.0888,0.1026)$} & 1.2914 & 1.5481 & 0.5299 & -0.1938 & 0.1162 & $1 / \sqrt{\pi}$ & 1.6069 & 0.8485 \\
{$[0.1026,0.2348)$} & 1.2914 & 1.5481 & 0.5299 & -0.0854 & -0.0604 & $1 / \sqrt{\pi}$ & 1.4837 & 1.0491 \\
{$[0.2348,0.5000)$} & 1.0000 & 1.0000 & 0.0000 & 0.0000 & 0.0000 & $1 / \sqrt{\pi}$ & 1.1284 & 0.7979 \\
\hline \hline
\end{tabular}

In Figure 2 we present the numerical solution at the final time $t=0.1$ of the simulation for the MR method for the different MHD variables. Similar results have been obtained with the FV method on a regular grid. Except for $v_{y}$, all the other variables are well represented by the used HLLD numerical flux at this resolution.

Figure 3 presents the time evolution of the divergence of the magnetic field with different MR thresholds values. Using the MR method with Harten's threshold strategy, the divergence of the magnetic field is well controlled with small values during the whole simulation.

For the MR/LT scheme, the enforcement of the update of $\psi$ due to the divergence correction in all RK stages improves the reduction of the divergence of the magnetic field, as shown in Table 4. This procedure is also required for the MR/CT scheme, as shown in Table 5. We observe that for the MR scheme (Table 3), without local time stepping or time step control, these extra computations do not reduce the divergence of the magnetic field. In all studied cases, the solution behavior or norms do not change significantly. These tables also present the compression of leaves, i.e., the compression of the cells that are effectively being used in the time evolution compared to the uniform mesh and the total memory used including the auxiliary cells to compute the flux and interpolations. We observe that in these threshold strategies the required additional memory is small compared to the improvement obtained for the divergence correction.

TABLE 3. Memory compression, $L^{2}$ error of density and $L_{\infty}$ error of the divergence of $\mathbf{B}$ for the MR method with constant and level dependent threshold.

\begin{tabular}{|c|c|c|c|c|c|}
\hline & \multicolumn{2}{|c|}{ Compression(\%) } & \multirow[t]{2}{*}{$\|\rho\|_{L^{2}}$} & \multirow{2}{*}{$\begin{array}{l}\max _{t, x, y} \\
|\nabla \cdot \mathbf{B}|\end{array}$} & \multirow[t]{2}{*}{$\|\nabla \cdot \mathbf{B}\|_{L^{2}}$} \\
\hline & Leaves & Memory & & & \\
\hline$\epsilon$ & \multicolumn{5}{|c|}{ method with $\epsilon^{\ell}=\epsilon$} \\
\hline 0.030 & 5.87 & 9.22 & 0.0088 & 1.40 & $2.88 \cdot 10^{-2}$ \\
\hline 0.003 & 13.87 & 20.35 & 0.0053 & $6.60 \cdot 10^{-5}$ & $9.85 \cdot 10^{-2}$ \\
\hline 0.001 & 16.11 & 23.08 & 0.0051 & $1.99 \cdot 10^{-5}$ & $1.39 \cdot 10^{-2}$ \\
\hline
\end{tabular}

\begin{tabular}{rrrrcr}
$\epsilon_{0}$ & \multicolumn{5}{c}{ method with variable $\epsilon^{\ell}$} \\
\hline 0.030 & 11.75 & 17.46 & $5.73 \cdot 10^{-3}$ & $9.66 \cdot 10^{-13}$ & $4.84 \cdot 10^{-14}$ \\
0.003 & 16.73 & 23.83 & $5.09 \cdot 10^{-3}$ & $11.37 \cdot 10^{-13}$ & $9.07 \cdot 10^{-14}$ \\
0.001 & 17.80 & 25.14 & $5.06 \cdot 10^{-3}$ & $10.80 \cdot 10^{-13}$ & $9.60 \cdot 10^{-14}$
\end{tabular}

NOTE: Computed with $\overline{\overline{\text { Runge-Kutta order } 2 \text { for MR. Parameters: } L=10, \mathrm{CFL}=0.4 \text {, final time }=0.1}}$ and $\gamma=\frac{5}{3}$. Memory and leaves compression used are related to the regular mesh $L=10$. 

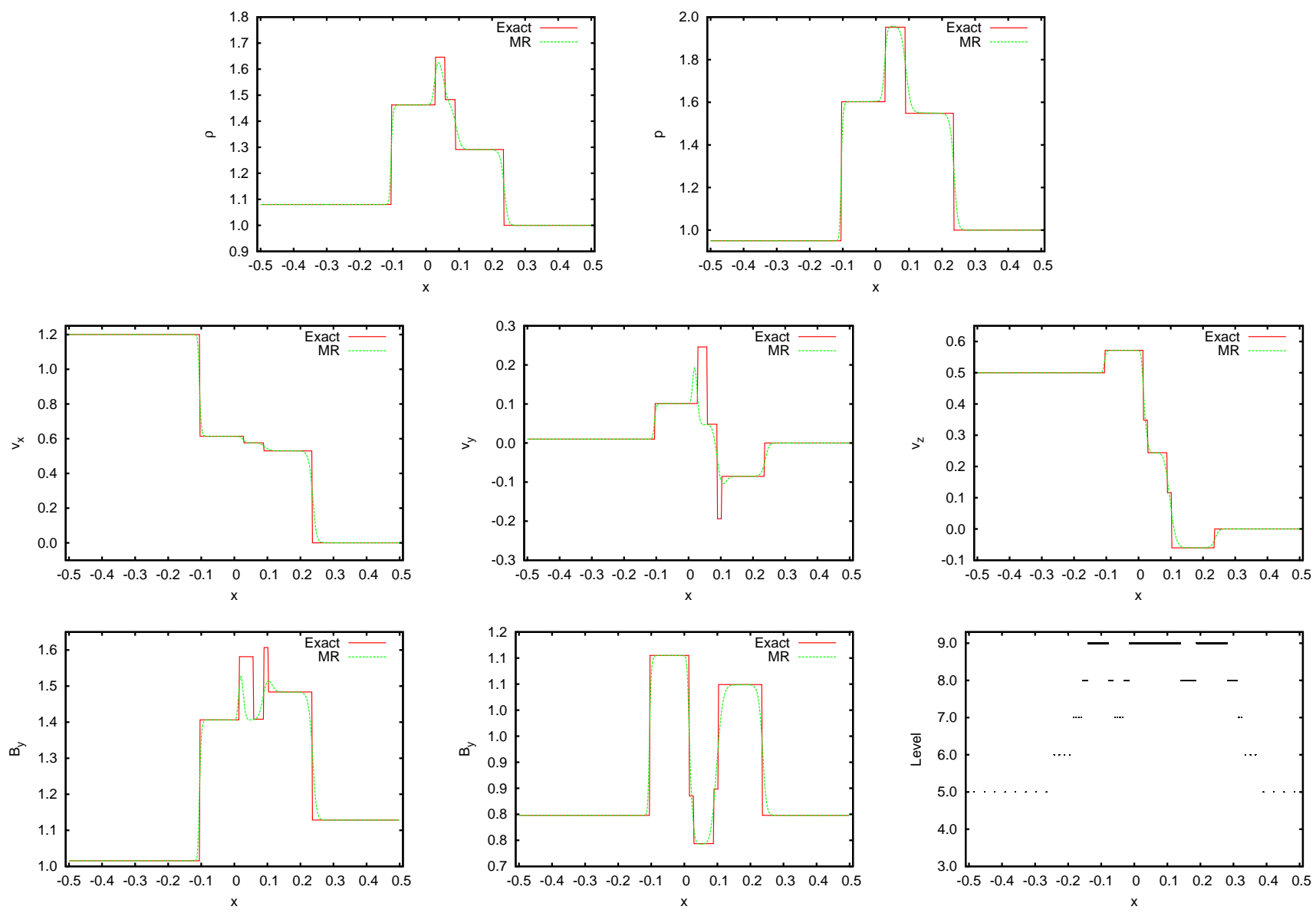

Figure 2. One-dimensional cuts of different MHD variables at time $t=0.1$ computed with MR using a corresponding finest underlying FV mesh of $512^{2}$ cells. The numerical parameters are: $\gamma=\frac{5}{3}, \mathrm{CFL}=0.4$, and $\frac{c_{h}}{c_{p}^{2}}=0.18$. A level dependent threshold is used with $\epsilon_{0}=0.001$. The corresponding adaptive mesh is shown on bottom, right. 

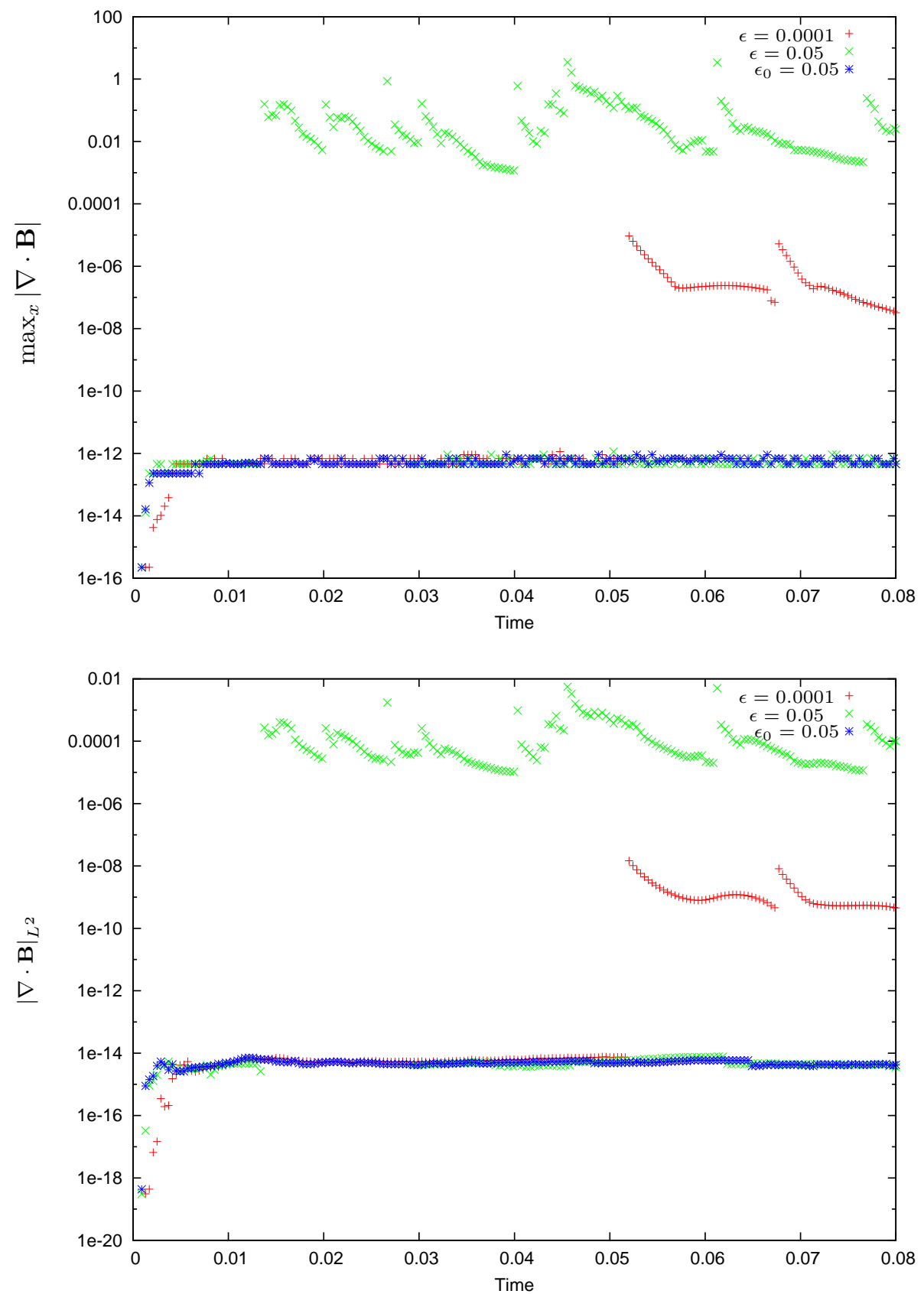

Figure 3. Time evolution of the $L^{2}$ (top) and $L_{\infty}$ (bottom) errors of $\nabla \cdot \mathbf{B}$ obtained with the MR method using a corresponding fine scale FV mesh of $512^{2}$. Further parameters are $\gamma=\frac{5}{3}$, $\mathrm{CFL}=0.4, \frac{c_{h}}{c_{p}}=1.0$. Results for the fixed threshold $\epsilon^{\ell}=\epsilon=0.0001$ are repesented in red, $\epsilon^{\ell}=\epsilon=0.05$ in green, and for the variable threshold with $\epsilon_{0}=0.05$ in blue. 
TABLE 4. Memory compression, $L^{2}$ error of density and $L_{\infty}$ error of the divergence of $\mathbf{B}$ for the MR/LT method with constant and level dependent threshold.

\begin{tabular}{|c|c|c|c|c|c|}
\hline & \multicolumn{2}{|c|}{ Compression (\%) } & \multirow[t]{2}{*}{$L^{2}(\rho)$} & \multirow{2}{*}{$\begin{array}{l}\max _{t, x, y} \\
|\nabla \cdot \mathbf{B}|\end{array}$} & \multirow[t]{2}{*}{$L^{2}(\nabla \cdot \mathbf{B})$} \\
\hline & Leaves & Memory & & & \\
\hline$\epsilon_{0}$ & \multicolumn{5}{|c|}{ method with variable $\epsilon^{\ell}$} \\
\hline 0.030 & 11.70 & 17.38 & 0.0059 & $6.20 \cdot 10^{-4}$ & $9.81 \cdot 10^{-7}$ \\
\hline 0.003 & 16.62 & 23.67 & 0.0051 & $6.20 \cdot 10^{-4}$ & $9.81 \cdot 10^{-7}$ \\
\hline 0.001 & 17.69 & 24.99 & 0.0050 & $6.20 \cdot 10^{-4}$ & $9.81 \cdot 10^{-7}$ \\
\hline
\end{tabular}

\begin{tabular}{rcrrrr}
$\epsilon_{0}$ & \multicolumn{5}{c}{ method with variable $\epsilon^{\ell}$ and extra $\nabla \cdot \mathbf{B}$ correction* $^{*}$} \\
\hline 0.030 & 11.70 & 17.38 & 0.0059 & $4.90 \cdot 10^{-8}$ & $1.09 \cdot 10^{-9}$ \\
0.003 & 16.62 & 23.67 & 0.0051 & $8.92 \cdot 10^{-8}$ & $2.78 \cdot 10^{-9}$ \\
0.001 & 17.69 & 24.99 & 0.0051 & $8.97 \cdot 10^{-8}$ & $3.10 \cdot 10^{-9}$
\end{tabular}

NOTE: Computed with Runge-Kutta order 2. Parameters: $L=10, \mathrm{CFL}=0.4$, final time $=0.1$ and $\gamma=\frac{5}{3}$. $*$ Inclusion of the $\psi$ update on each stage of the RK method. Memory and leaves compression used are related to the regular mesh $L=10$.

TABLE 5. Memory compression, $L^{2}$ error of density and $L_{\infty}$ error of the divergence of $\mathbf{B}$ for the MR/CT method with constant and level dependent threshold.

\begin{tabular}{|c|c|c|c|c|c|}
\hline & \multicolumn{2}{|c|}{ Compression $(\%)$} & \multirow[t]{2}{*}{$\|\rho\|_{L^{2}}$} & \multirow{2}{*}{$\begin{array}{l}\max _{t, x, y} \\
|\nabla \cdot \mathbf{B}|\end{array}$} & \multirow[t]{2}{*}{$\|\nabla \cdot \mathbf{B}\|_{L^{2}}$} \\
\hline & Leaves & Memory & & & \\
\hline$\epsilon_{0}$ & \multicolumn{5}{|c|}{ method with $\epsilon^{\ell}$ and extra $\nabla \cdot \mathbf{B}$ correction* $^{*}$} \\
\hline 0.030 & 11.11 & 16.47 & 0.0057 & $9.1 \cdot 10^{-13}$ & $5.5 \cdot 10^{-14}$ \\
\hline 0.003 & 15.21 & 21.67 & 0.0051 & $4.4 \cdot 10^{-10}$ & $1.9 \cdot 10^{-11}$ \\
\hline 0.001 & 16.14 & 22.82 & 0.0051 & $4.5 \cdot 10^{-10}$ & $2.4 \cdot 10^{-11}$ \\
\hline
\end{tabular}

NOTE: Computed with Re $\overline{\overline{u n g e-K u t t a} \text { order } 2(3) . \text { Parameters: } L=10, \mathrm{CFL}(0)=0.4, \text { final time }}=0.1, \delta_{\text {desired }}=0.001$ and $\gamma=\frac{5}{3}$. ${ }^{*}$ Inclusion of the $\psi$ update on each stage of the RK method. Memory and leaves compression used are related to the regular mesh $L=10$. 


\section{FINAL REMARKS}

In the current study we presented a new adaptive MR code for ideal MHD equations coupled with a divergence cleaning technique to impose the incompressibility of the magnetic field on locally refined adaptive mesh and performed numerical experiments in two space dimensions.

For constant threshold values $\epsilon$ the magnetic fields do not remain divergence free, even larger values of divergence do occur compared to the results obtained using Harten's threshold strategy. Using the latter strategy, the divergence of the magnetic field is well controlled and remains small during the simulation. Hence, we conclude that Harten's thresholding rule yields reasonable results for the current example. Applying the same thresholding rule to MR/LT and MR/CT we found that the divergence is larger than for classical MR.

We concluded from this study that new strategies for applying divergence techniques are needed when applying adaptive time stepping methods. Investigation of such techniques will be the aim of future work together with the implementation in three space dimensions.

\section{ACKNOWLEDGMENTS}

M. O. Domingues, S. M. Gomes and O. Mendes thankfully acknowledge financial support from CAPES (grants 86/201029), CNPq (grants 483226/2011 - 4,306828/2010 - 3,307511/2010 - 3, 486165/2006 - 0,305274/2009-0), Ecole Centrale de Marseille (ECM), and FAPESP (grants 2012/06577 - 5,2012/072812 - 2,2007/07723 - 7). A. Gomes thankfully acknowledges financial support for her Master Degree from CNPq. O. Mendes acknowledges financial support from PGGES/PROEX-CAPES. M. O Domingues and K. Schneider thank the ANR projects M2TFP and SiCOMHD for financial support. The authors thank CEMRACS 2012 and CIRM for the hospitality and the comments of the reviewers that contributed to the improvement of this paper. We are grateful to Dominique Fougère, Marie G. Dejean and Varlei E. Menconi (FAPESP grants 2008/09736-1 and MCTI/INPE-PCI 312486/2012-0) for their helpful computational assistance.

\section{REFERENCES}

[1] F. Assous, P. Degond, E. Heintze, P.A. Raviart, and J. Segre. On a finite-element method for solving the three-dimensional Maxwell equations. J. Comput. Phys., 109(2):222-237, 1993.

[2] Dinshaw S. Balsara. Divergence-free reconstruction of magnetic fields and WENO schemes for magnetohydrodynamics. $J$. Comput. Phys., 228:5040-5056, 2009.

[3] J. U. Brackbill and D. C. Barnes. Note: The effect of nonzero $\nabla \cdot \mathbf{B}$ on the numerical solution of the magnetohydrodynamic equations. J. Comput. Phys., 35(3):426-430, 1980.

[4] A. Cohen, S. M. Kaber, S. Müller, and M. Postel. Fully adaptive multiresolution finite volume schemes for conservation laws. Math. Comput., 72(241):183-225, 2003.

[5] A. Dedner, F. Kemm, D. Kröner, C.-D. Munz, T.Schnitzer, and M. Wesenberg. Hyperbolic divergence cleaning for the MHD equations. J. Comput. Phys., 175:645-673, 2002.

[6] A. Dedner, C. Rohde, and M. Wesenberg. A new approach to divergence cleaning in magnetohydrodynamic simulations. In ThomasY. Hou and Eitan Tadmor, editors, Hyperbolic Problems: Theory, Numerics, Applications, pages 509-518. Springer Berlin Heidelberg, 2003.

[7] B. Di Pierro. Méthode d'annulation de la divergence pour les EDP hyperboliques application aux équations de la magnétohydrodynamique. Final project Master Course, Université de Provence, Marseille, France, 2009. (unpublished, in French).

[8] M. O. Domingues, S. M. Gomes, O. Roussel, and K. Schneider. An adaptive multiresolution scheme with local time stepping for evolutionary PDEs. J. Comput. Phys., 227(8):3758-3780, 2008.

[9] M. O. Domingues, S. M. Gomes, O. Roussel, and K. Schneider. Space-time adaptive multiresolution methods for hyperbolic conservation laws: Applications to compressible Euler equations. Appl. Numer. Math., 59:2303-2311, 2009.

[10] M. O. Domingues, S. M. Gomes, O. Roussel, and K. Schneider. Adaptive multiresolution methods. ESAIM Proc., 34:1-96, 2011.

[11] M. O. Domingues, O. Roussel, and K. Schneider. An adaptive multiresolution method for parabolic PDEs with time-step control. Int. J. Numer. Meth. Engng., 78:652-670, 2009.

[12] A. K. F. Gomes. Análise Multirresolução adaptativa no contexto da resolução numérica de um modelo de Magnetohidrodinâmica ideal. Master's thesis, Instituto Nacional de Pesquisas Espaciais (INPE), São José dos Campos, 2012-09-13 2012. (sid.inpe.br/mtc-m19/2012/08.10.15.02-TDI, http://urlib.net/8JMKD3MGP7W/3CE6FSE, in Portuguese).

[13] A. Harten. Multiresolution Algorithms for the Numerical Solution of Hyperbolic Conservation Laws. Commun. Pur. Appl. Math., 48:1305-1342, 1995. 
[14] A. Harten. Multiresolution representation of data: a general framework. SIAM J. Numer. Anal., 33(3):385-394, 1996.

[15] S. S. Komissarov, M. Barkov, and M. Lyutikov. Tearing instability in relativistic magnetically dominated plasmas. Mon. Not. R. Astron. Soc, 374:415-426, 2007.

[16] T. Mioshi and K. A. Kusano. A multi-state HLL approximate Riemann solver for ideal magnetohydrodynamics. J. Comput. Phys., 208:315-344, 2005.

[17] S. Müller. Adaptive multiscale schemes for conservation laws, volume 27 of Lectures Notes in Computational Science and Engineering. Springer, Heidelberg, 2003.

[18] C.-D. Munz, P. Ommes, R. Schneider, E. Sonnendrücker, and U. Voss. Divergence corrections techiniques for Maxwell solvers based on a hyperbolic model. J. Comput. Phys., 161(2):484, 2000.

[19] K. G. Powell. An approximate Riemann solver for magnetohydrodynamics (That works in more than one dimension). Technical report.

[20] K. G. Powell, P. L. Roe, T. J. Linde, T. I. Gombosi, and D. L. D. Zeeuw. A solution-adaptative upwind scheme for ideal magnetohydrodynamics. J. Comput. Phys., 154:284-309, 1999.

[21] O. Rousell, K. Schneider, A. Tsigulin, and H. Bockhorn. A conservative fully adaptative multiresolution algorithm for parabolic PDEs. J. Comput. Phys., 188:493-523, 2003.

[22] M. Torrilhon. Uniqueness conditions for Riemann problems of ideal magnetohydrodynamics. J. Plasma Phys., 96:253-276, 2003.

[23] G. Tóth. The $\nabla \cdot \mathbf{B}$ constraint in shock-capturing magnetohydrodynamics codes. J. Comput. Phys., 161:605-652, 2000.

[24] T. S. Tricco and D. J. Price. Constrained hyperbolic divergence cleaning for smoothed particle magnetohydrodynamics. J. Comput. Phys., 231(21):7214-7236, 2012. 